\title{
UAV-BASED DETECTION OF UNKNOWN RADIOACTIVE BIOMASS DEPOSITS IN CHERNOBYL'S EXCLUSION ZONE
}

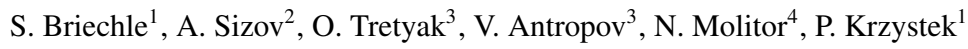 \\ ${ }^{1}$ Munich University of Applied Sciences, Munich, Germany - (sebastian.briechle, peter.krzystek)@hm.edu \\ ${ }^{2}$ Institute for Nuclear Safety Problems for Nuclear Power Plants, Kiev, Ukraine - a.sizov@gmail.com \\ ${ }^{3}$ State Central Enterprise for Radioactive Waste Management, Kiev, Ukraine - tretyak.og@gmail.com, valant@ voliacable.com \\ ${ }^{4}$ Plejades GmbH, Griesheim, Germany - norbertmolitor@pleja.de
}

Commission II, ICWG I/II

KEY WORDS: UAV, LiDAR, gamma spectrometry, 3D vegetation mapping, biomass, machine learning

\begin{abstract}
:
Shortly after the explosion of the Chernobyl nuclear power plant (ChNPP) in 1986, radioactive fall-out and contaminated trees (socalled Red Forest) were buried in the Chernobyl Exclusion Zone (ChEZ). These days, exact locations of the buried contaminated material are needed. Moreover, 3D vegetation maps are necessary to simulate the impact of tornados and forest fire. After 30 years, some of the so-called trenches and clamps are visible. However, some of them are overgrown and have slightly settled in the centimeter and decimeter range. This paper presents a pipeline that comprises $3 \mathrm{D}$ vegetation mapping and machine learning methods to precisely map trenches and clamps from remote sensing data. The dataset for our experiments consists of UAV-based LiDAR data, multi-spectral data, and aerial gamma-spectrometry data. Depending on the study areas overall accuracies ranging from $95.6 \%$ to $99.0 \%$ were reached for the classification of radioactive deposits. Our first results demonstrate an accurate and reliable UAV-based detection of unknown radioactive biomass deposits in the ChEZ.
\end{abstract}

\section{INTRODUCTION}

In the first months after the Chernobyl accident large parts of contaminated material resulting from clean-up operations were buried into nine radioactive waste temporary storage places (RWTSP). On the one hand, these clean-up measures resulted in a decrease of the external exposure dose rate by a factor of about 10. Parts of the RWTSP were also re-vegetated in order to stabilize the surface covers and to reduce the effect of re-suspension of contaminated dust particles by wind. On the other hand, however, these clean-up operations led to a higher risk of groundwater contamination. These RWTSP were created in the vicinity of the ChNPP and contain about 1000 excavated trenches and/or clamps in which radioactive material was buried and covered with a clean soil layer (Molitor et al., 2017b). Unfortunately, a comprehensive and complete documentation of the volumes, the radionuclide inventories, the exact positions, and the exact total number of trenches and clamps is not available. In order to justify and substantiate action plans to mitigate actual and future hazards, it is extremely important to have appropriate up-to-date descriptions and evaluations on the current radiological situation and its evolution.

Since the explosion of the ChNPP a lot of research investigation from different special fields have been carried out in the ChEZ. To name just a few: Kashparov et al. (2012) present studies on the radionuclide behavior in the environment after the disposal of radioactive waste into shallow subsurface storages. The main results describe the biogenic migration of radionuclides from subsurface storages to plants. Bugai et al. (2012) focused on the hydrogeological characterization and groundwater transport modeling to describe the migration of radionuclides over a 16-year period (1986-2002). Besides these research studies, the European Union financed a project called
"Support to radioactive waste management in Ukraine" (EU, 2014-2017). One purpose of this project was the investigation of radioactive waste burial. Partially, the RWTSP have already been well investigated. Though, some other RWTSP do not have such detailed information about the location and radioactivity of inventory - these are subject for ongoing investigation efforts. In 2015, airborne geophysical surveys proved that the mapping of burials - especially in low vegetated areas - is possible using high-resolution gamma field radiation measurements. The results prove that the local gamma ray intensity significantly exceeds the level of the total background radiation in contaminated areas. In vegetated areas, the biomass significantly distorts the measurements because the radiation of the vegetation can not be taken into account during the calibration process (Zabulonov et al., 2015).

The experiments with the gamma spectrometer conducted so far in the forested areas of ChEZ reveal that the interpretation of the spectrometer measurement signal is difficult and not yet solved. Thus, other sensor systems are to be envisaged supplementary to detect buried contaminated material. As these deposits can be characterized by slight height anomalies of the ground (trenches and clamps) the idea is to advantageously use an UAV-based LiDAR system: On the one hand, this measurement technique operates at a sufficient safety distance, which is of prime importance considering the study area. On the other hand, UAV-based LiDAR permits a precise $3 \mathrm{D}$ reconstruction of the tree landscape as well as the detection of small terrain height anomalies. It even allows the creation of 3-dimensional contamination maps by taking into account the tree specific radiation. In non-forested areas some of the known deposits are covered with low vegetation that - compared to the neighbourhood shows anomalies w.r.t the species and the green tone. Hence, we also used a UAV-mounted high resolution multi-spectral 
camera to collect image data in the visible and near-infrared (NIR) spectrum that provides additional information for the classification process. To sum it up, the key idea of this research is to combine UAV-based LiDAR, multi-spectral images and airborne gamma spectrometer measurements to automatically map unknown buried radioactive deposits in the ChEZ.

In the following sections we address (ii) the drone systems, the sensors and the pre-processing of the data, (iii) the entire processing pipeline including the 3D vegetation mapping and the classification scheme for the radioactive burials, (iv) the experiments and results, (v) discuss our results and (vi) conclude and give an outlook to potential future research topics.

\section{MATERIAL}

\subsection{UAV systems}

Persuading results from UAV surveys start with the selection of the appropriate sensors to perform the requested task. Nevertheless, the capability of the carrier system will decide on the performance of the survey both in terms of quality and quantity. To achieve our objectives we relied on two different UAVs. For the LiDAR measurements an octocopter was available that was developed by a team of the department of nuclear physics technologies of the NASU Institute of Environment Geochemistry (NASU: National Academy of Sciences of Ukraine). This octocopter was already used for the airborne geophysical surveys mentioned above. As carrier for the multi-spectral camera we used the Quantum Trinity VTOL (vertical take-off and landing) fixed-wing system. The hybrid concept of this VTOL drone combines the advantages of multi-copters and fixed-wing drones. Using multiple propeller arrays or tilt rotors, it can take off and land like a rotary wing drone. Once airborne, it can transition to horizontal flight and work as a fixed-wing drone.

\subsection{LiDAR data}

As LiDAR system we used the YellowScan Mapper laserscanner mounted on the octocopter carrying the payload of $2.2 \mathrm{~kg}$ (excl. copter batteries) at a maximum mission time of around 20 minutes. In order to get flight trajectories with centimeter precision a GNSS base station was set up to collect simultaneous measurements that were used in DPGS processing. Seven flights were conducted in four selected areas of ChEZ in November 2017 in leaf-off situation. The measurement rate was $18.5 \mathrm{kHz}$, the flights were realized at a speed of $4-7 \mathrm{~m} / \mathrm{s}$ and an altitude of $50 \mathrm{~m}$ resulting in a nominal point density of $25-40$ points $/ \mathrm{m}^{2}$ (side lap $50 \%$ ). The boresight parameters of the laserscanner provided by the manufacturer were checked using data of a special calibration flight over a building and were fully verified in the subsequent strip alignment. All the strips of a flight mission were aligned to achieve a consistent LiDAR point cloud using the software package BayesStripAlign from BayesMap Solutions. At average, the mean discrepancies between the neighbouring strips were around $5 \mathrm{~cm}$. This means that the boresight parameters had been successfully adjusted and the strip trajectories appropriately mapped. An absolute 3D georeferencing was achieved by fitting the LiDAR point clouds to the enclosing polygons of buildings. Finally, ground points were filtered from the LiDAR point clouds and were subsequently interpolated into a digital terrain model (DTM) grid with a grid size of $0.5 \mathrm{~m}$.

\subsection{Multi-spectral data}

To capture multi-spectral data we used the Tetracam ADC Snap multi-spectral camera mounted on the Quantum Trinity VTOL system. The ADC Snap camera captures light wavelengths in three bands between $520 \mathrm{~nm}$ and $920 \mathrm{~nm}$ (Green, Red, NIR). The flight duration of the Trinity drone was around $50 \mathrm{~min}$ utes at a mean speed of $17 \mathrm{~m} / \mathrm{s}$ (flying altitude $130 \mathrm{~m}$, side lap $40 \%$, end lap $80 \%$ ). All flights were carried out in fully automatic mode to avoid the direct trespassing of contaminated areas. Dense photogrammetric point clouds $\left(15\right.$ points $\left./ \mathrm{m}^{2}\right)$ and orthomosaics (ground sampling distance $8 \mathrm{~cm}$ ) were generated from the multi-spectral images using standard structure-from-motion (SfM) Software. Due to missing ground control points (GCPs) in a few inaccessible areas two photogrammetric point clouds were registered to the LiDAR data by manual measurements of GCPs with a standard deviation of $70 \mathrm{~cm}$.

\subsection{Gamma spectrometry data}

In addition to the LiDAR data and the multi-spectral images, measurements of an aerial gamma-ray spectrometer survey were available as selective data. The UAV-based aero-gamma spectrometry system (see Figure 1) enables high-resolution mapping of radiation contamination without any risk to human health. The spectrometer data were collected by the airborne gamma spectrometric complex (SC) "ASPEK". The on-board unit is intended to perform geo-referenced measurements of the gamma radiation spectra as well as altitude, pressure and temperature measurements. The spectrometer consists of five blocks of gamma radiation detectors based on thallium-doped sodium iodide scintillator crystals with a size of $63 \times 63 \mathrm{~mm}$ (Zabulonov et al., 2015). These crystals emit light as soon as gamma rays interact with the atoms in the crystals. The intensity of the produced light can be measured and is proportional to the energy deposited by the gamma rays (Melcher, 2000). All blocks work synchronously and the signals of all detectors are summarized. The measurement system allows real-time control over the unit sensitivity by turning on/off single detector blocks - depending on the required sensitivity. With a total weight of about $7.5 \mathrm{~kg}$ (excl. batteries) and a dimension of $300 \mathrm{~mm} \times 300 \mathrm{~mm}$ the spectrometer can be installed on an octocopter (Zabulonov et al., 2017).

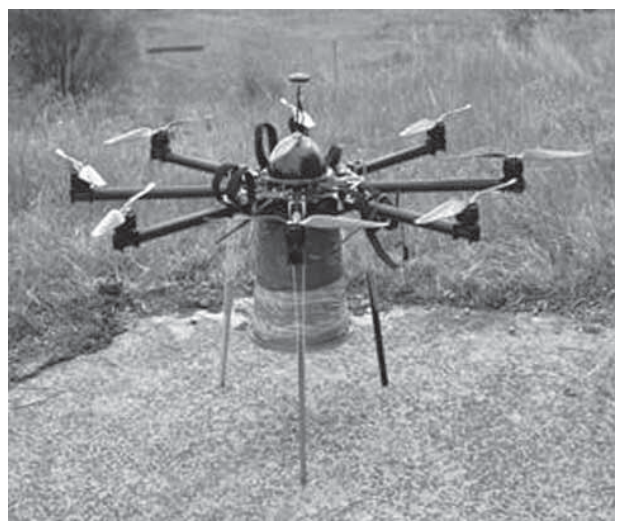

Figure 1. UAV-mounted gamma spectrometric complex (Zabulonov et al., 2017)

The flights were performed in parallel tracks with a track-to-track distance of $100 \mathrm{~m}$. The average altitude was $30 \mathrm{~m}$ at an average flight speed of $5 \mathrm{~m} / \mathrm{s}$ and a measurement interval of $1 \mathrm{~s}$. The high 


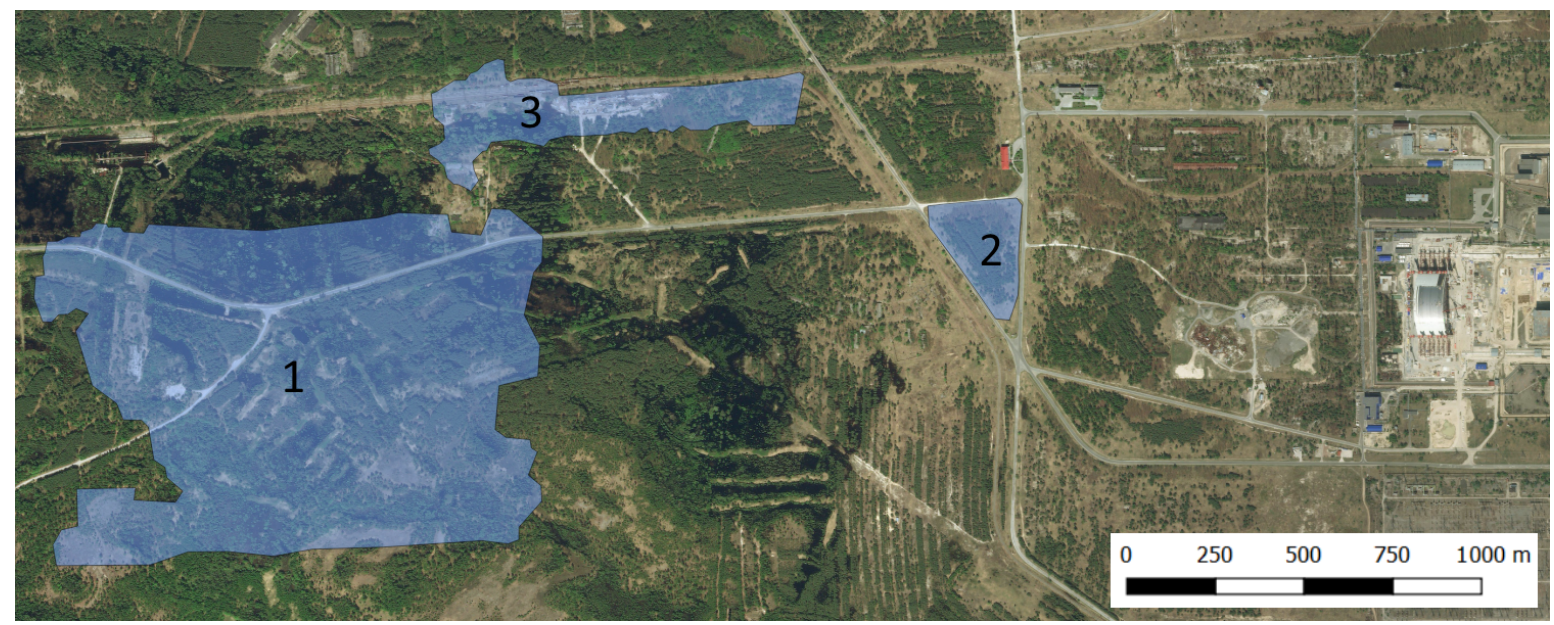

Figure 2. Locations of the three study areas in the ChEZ (source: bing map @Microsoft Corporation)

payload of the detector array does not allow any physical collimation. This fact results in a spectrometer measurement angle of 120 degrees with a footprint of around $100 \mathrm{~m}$. The pre-processing of the spectrometry data contained the following steps: First, the total gamma intensity (gamma_total) was computed. In a second step, the background gamma intensity was calculated using a sliding average method. Finally, the local component of the gamma intensity (gamma_local) was derived as the difference of the total gamma intensity and the background gamma intensity (Zabulonov et al., 2015). It needs to be mentioned that the measured signal is the sum of different signals, i.e. the natural radiation from space and ground, the radiation from close-range contaminated vegetation, the radiation from fallout on the ground, and from buried biomass

\subsection{Study areas}

Three different study areas are presented in this paper (see Figure 2). Study area "Yanov Station 3.3/3.5/3.7" (size around 1 $\mathrm{km}^{2}$ ) was just captured with the multi-spectral camera. Most of the clamps are clearly visible because they are not covered with high vegetation. In the second study area "Red Forest 2.5 " (area size 6 ha), dense vegetation (birches and pines with a height up to 21 metres) covers the ground and makes it impossible to detect trenches and clamps just using an airborne photogrammetric sensor. In this case, the LiDAR sensor is superior because of the penetration of the laser beams to the ground. Study area "Yanov Station 3.3" (area size 15 ha) is the former railway freight terminal which is partly overgrown with birches and pines with a height up to 27 metres. In this area, no photogrammetric data are available.

\section{METHODS}

Depending on the tree species parts of the contaminated material are accumulated in the above-ground biomass. The radioactive deposits are characterized by slight ground settlements or elevations, vegetation anomalies and a higher rate of gamma decays. Thus, the key idea is to classify the deposit areas from salient features calculated from the bare ground height, the vegetation, the gamma radiation and the reflection data acquired in the visible and NIR channels.

\subsection{D vegetation mapping}

Based on the normalized cut algorithm single trees are automatically segmented from the LiDAR data resulting in tree position, tree height, crown volume and crown base height for each tree (Reitberger et al., 2009). The tree segmentation is evaluated by visual interpretation to find the best Ncut threshold since no tree reference data are available. Subsequently, the single trees are classified w.r.t. to the two main tree species birch (Betula pubescens) and pine (Pinus sylvestris) using a Random Forest (RF) classifier. The feature set (48 features) comprises radiometric and geometric features, e.g. height dependent and density dependent features, the crown shape and the mean intensity of laser points of a single tree per height layer.

Since no destructive sampling of reference trees is available we need to estimate the tree biomass solely from allometric equations using the tree height and tree species. First, we calculate the diameter at breast height $(\mathrm{DBH})$ from the tree height (Widlowski et al., 2003). Next, we convert the DBH into the total above-ground biomass using the equations in Repola et al. (2007).

\subsection{Detection of trenches and clamps}

In order to automatically detect trenches and clamps it is necessary to generate meaningful features from the available data and apply the classification based upon a well-defined evaluation strategy.

\subsubsection{Feature description}

A meaningful feature to describe terrain height anomalies is the normalized height. For every DTM grid position, the corresponding absolute terrain height is reduced by the height trend (mean height in a surrounding quadratic area of $50 \times 50 \mathrm{~m}$ ). Since contaminated areas are supposed to be characterized by vegetation anomalies multiple features are calculated to describe the vegetation. One set of features are the so-called LiDAR metrics (Næsset, 2004). These height- and density-dependant features are calculated for eight different height layers $(0-1.5 \mathrm{~m}, 1.5-5 \mathrm{~m}, 5-12$ $\mathrm{m},>12 \mathrm{~m},>2 \mathrm{~m}, 0.5-2 \mathrm{~m}, 0.5-5 \mathrm{~m},>0 \mathrm{~m}$ ) for a cell size of $5 \mathrm{x}$ $5 \mathrm{~m}$. Finally, the metrics are resampled to the DTM grid size by bi-linear interpolation. Another feature set describing the vegetation comprises 14 so-called "tree features" (see Table 1) which are calculated for every grid point of the DTM. The close-up trees 
in a circular area are detected by applying a range search within a radius of $30 \mathrm{~m}$. Available features that characterize the gamma radiation are the total intensity and the local component of the gamma spectrometry measurements. These data are available as single measurements (point distance around $5 \mathrm{~m}$ ) along the UAV flight tracks and are bi-linearly interpolated on the DTM grid. Additionally, multi-spectral data (ortho-mosaic) are available for study area 1 . The data of the three channels (Green, Red, NIR) are also bi-linearly interpolated on the DTM grid. All in all, the generated features can be grouped into five feature subsets (see Table 2). The total feature sets for the particular experiments are individually generated w.r.t. the characteristics of the study areas and the availability of remote sensing data.

Table 1. Definition of tree features (tf)

\begin{tabular}{|c|l|}
\hline tf\# & Definition \\
\hline \hline 1 & number of trees \\
2 & mean tree height [m] \\
3 & std of tree height [m] \\
4 & mean CB_height [m] \\
5 & std of CB_height [m] \\
6 & pine tree ratio (\# pine trees / \# all trees) \\
7 & sum of crown volumes $\left[\mathrm{m}^{3}\right]$ \\
8 & mean crown volume $\left[\mathrm{m}^{3}\right]$ \\
9 & std of crown volume $\left[\mathrm{m}^{3}\right]$ \\
10 & mean DBH [cm] \\
11 & std of DBH [cm] \\
12 & sum of above-ground biomass $[\mathrm{kg}]$ \\
13 & mean above-ground biomass $[\mathrm{kg}]$ \\
14 & std of above-ground biomass $[\mathrm{kg}]$ \\
\hline \multicolumn{2}{|}{}
\end{tabular}

Table 2. Available feature subsets in the study areas

\begin{tabular}{|l|c|c|c|}
\hline study area & 1 & 2 & 3 \\
\hline \hline normalized height & $\mathrm{X}^{1}$ & $\mathrm{X}$ & $\mathrm{X}$ \\
\hline LiDAR metrics & $\mathrm{X}^{\mathrm{I}}$ & $\mathrm{X}$ & \\
\hline tree features & & $\mathrm{X}$ & \\
\hline gamma spectrometry features & $\mathrm{X}$ & $\mathrm{X}$ & $\mathrm{X}$ \\
\hline multi-spectral features & $\mathrm{X}$ & & \\
\hline
\end{tabular}

\subsubsection{Classification and evaluation strategy}

Prior to the RF classification, training and test areas are manually defined and labeled according to their classes by visual interpretation of the datasets. Partially available RWTSP reference data also support the labeling. The training set is balanced and highly correlated redundant features are removed from the feature set to avoid a deterioration of the classification result. First, we calculate the correlation coefficient from the covariance matrix of all features. Second, we eliminate features with a correlation coefficient larger than 0.75 . A RF classifier is trained including a recursive feature elimination (RFE) to optimize the feature set by discarding non-important features. The classifier is evaluated by the overall accuracy and kappa values by a repeated 5 -fold cross-validation. Moreover, the mean decrease in accuracy (proportion of observations that are incorrectly classified by removing the feature) is used to evaluate the relevance of the individual features. The class predictions for the test data are estimated and the prediction quality is checked using the overall accuracy, precision and recall. Finally, the class probabilities beyond a certain threshold (i.e. 95\%) are visualized for the particular study areas.

\footnotetext{
${ }^{1}$ For study area 1 (Yanov Station 3.3/3.5/3.7), the normalized height and the "LiDAR" metrics are calculated from the photogrammetric point cloud due to missing LiDAR data.
}

\section{EXPERIMENTS}

\subsection{Vegetation mapping and biomass estimation}

Based on the segmentation of single trees the two tree species (birch, pine) were classified using a RF classifier. The classifier was trained using a reference dataset in study area 2 consisting of 216 manually labeled, well-balanced tree species and a sample ratio of 0.7 (70\% for training, $30 \%$ for test). A 5-fold cross-validation showed an overall accuracy of $95.4 \%$, a kappa value of $91.4 \%$, a precision of $94.3 \%$, and a recall of $97.1 \%$. After a feature relevance assessment the mean intensity of the tree crowns turned out to be the most relevant feature.

The DBH parameters were estimated from the tree heights using allometric functions and were subsequently used to estimate the total above-ground biomass (see 3.1). Figure 3 shows the segmented trees for the study area Red Forest 2.5 colored in dependence on the tree species (a) and on their total above-ground biomass (b), respectively.

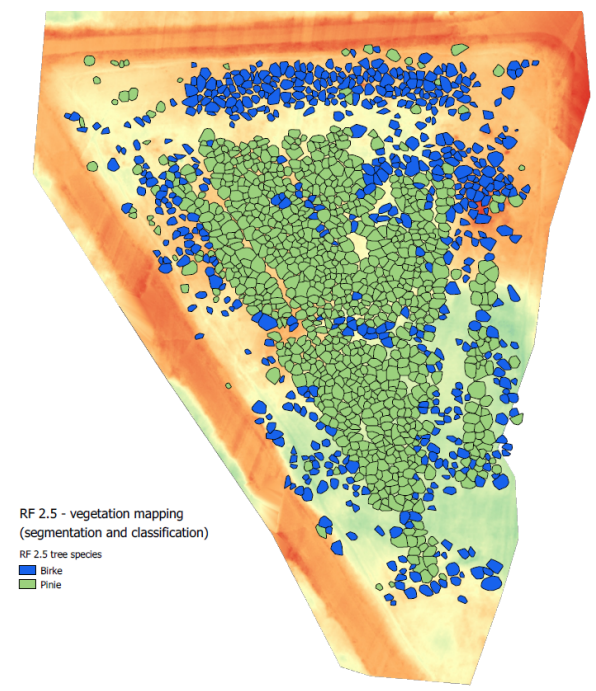

(a)

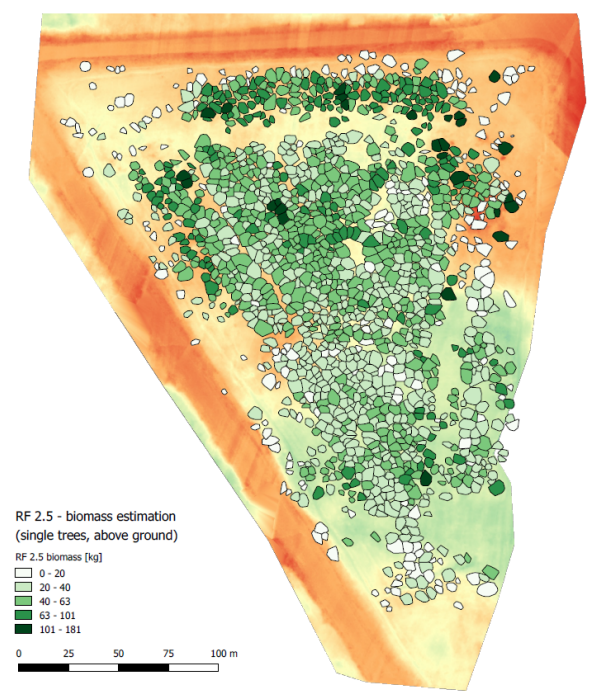

(b)

Figure 3. Study area 2: (a) Tree species classification result; (b) Biomass estimation result 


\subsection{Detection of trenches and clamps}

The individual feature subsets in the study areas that were used as input for the feature selection process are depicted in Table 2. Table 3 gives an overview of the number of effectively applied features in the classification process (used \#feat), the class names, the number of training and test samples, the 5-fold crossvalidation results of the training dataset and the results of the class prediction of the test data $(\mathrm{OA}=$ overall accuracy, prec. $=$ precision, rec. = recall). In the following sub-sections, the results of the different study areas are presented in detail.

\subsubsection{Study area 1: Yanov Station 3.3/3.5/3.7}

During the feature selection the gamma spectrometry features, the green channel as well as huge parts of the "LiDAR" metrics were removed. The RFE process resulted in 16 remaining features out of 22 uncorrelated features. The feature importance depending on the mean decrease in accuracy showed that - as expected - the normalized height and the NIR channel are by far the most relevant features. Class predictions and class probabilities were calculated for the test area (Figure 4a). The prediction result of class probability values of more than $95 \%$ for the class "clamps" are presented in Figure $4 \mathrm{~b}$. The detected clamps in this study area have already been known to the ChEZ authorities. These relatively high, non-vegetated clamps can partly be seen from the non-contaminated roads and even in satellite images. Nevertheless, the results $(\mathrm{OA}=95.6 \%$, kappa $=89.5 \%$, precision $=93.6 \%$, recall $=91.7 \%$; see Table 3 ) show that our method is feasible to detect this kind of clamps that are solely captured by multi-spectral cameras.

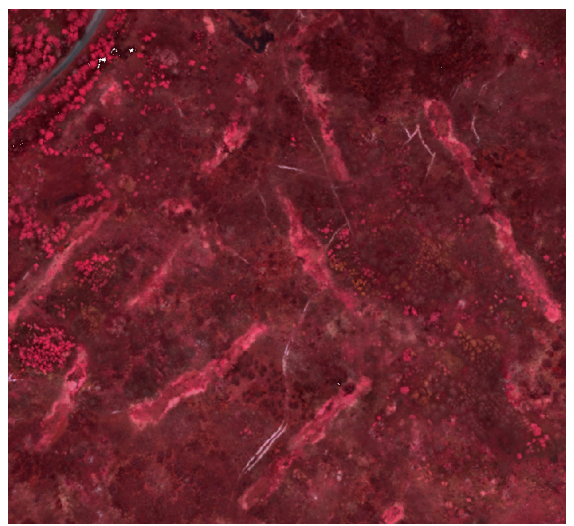

(a)

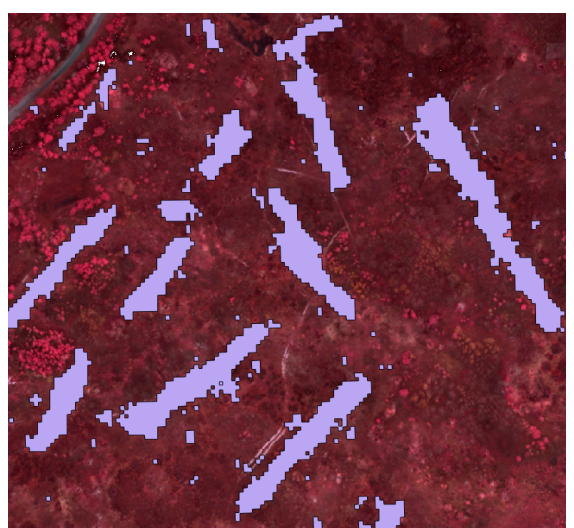

(b)

Figure 4. Study area 1: (a) Test area; (b) Classification result for class "clamp", class probability values $>95 \%$

\subsubsection{Study area 2: Red Forest 2.5}

In the area Red Forest 2.5, the RFE dropped 5 of the 17 uncorrelated features. Four LiDAR metrics as well as the gamma local component were removed during this step. It is not surprising that the normalized height was again the most important feature. Besides eight of the LiDAR metrics, three tree features remained in the feature set. In this study area with dense vegetation, two of the tree features are even ranked under the top five. Obviously, vegetation anomalies occur in the trench areas since the standard deviations of the crown volume, the above-ground biomass, and the DBH turned out as prominent features. The classification result is revealed in an excellent accuracy $(\mathrm{OA}=99.0 \%$, $\mathrm{kappa}=98.0 \%$, precision $=99.4 \%$, recall $=98.6 \%$; see Table 3 ). The filtered DTM (a) as well as the prediction result of the study area 2 (b) are presented in Figure 5. In the area Red Forest 2.5, four so far unknown trenches overgrown with quite dense vegetation were detected. The length of these trenches ranges between $50 \mathrm{~m}$ and $100 \mathrm{~m}$ with a width up to $5 \mathrm{~m}$ and a depth up to $1.5 \mathrm{~m}$.

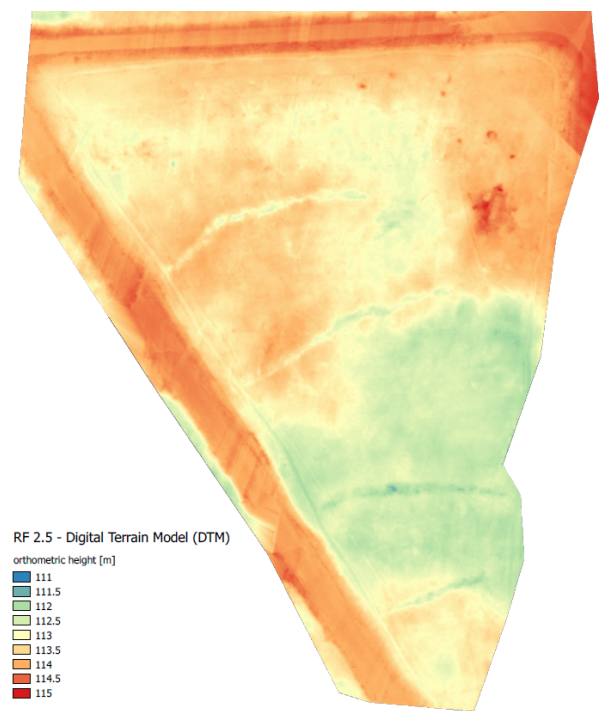

(a)

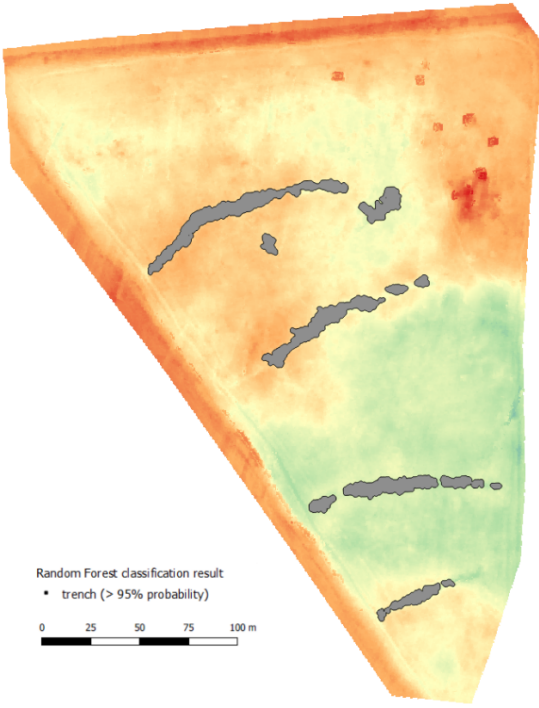

(b)

Figure 5. Study area 2: (a) Filtered DTM; (b) Classification result for class "trench", class probability values $>95 \%$ (b) 


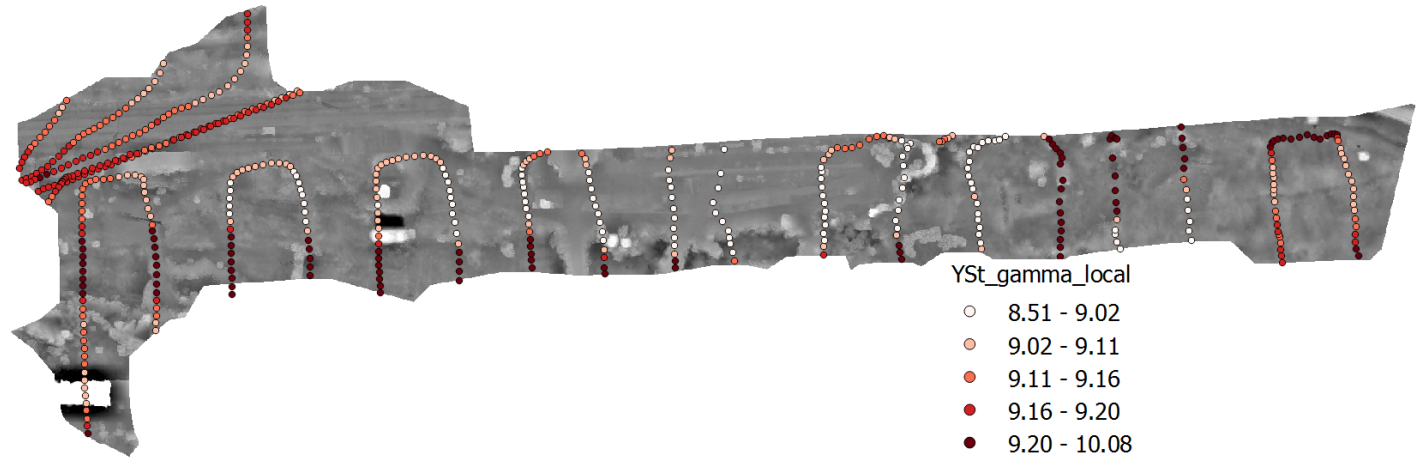

(a)

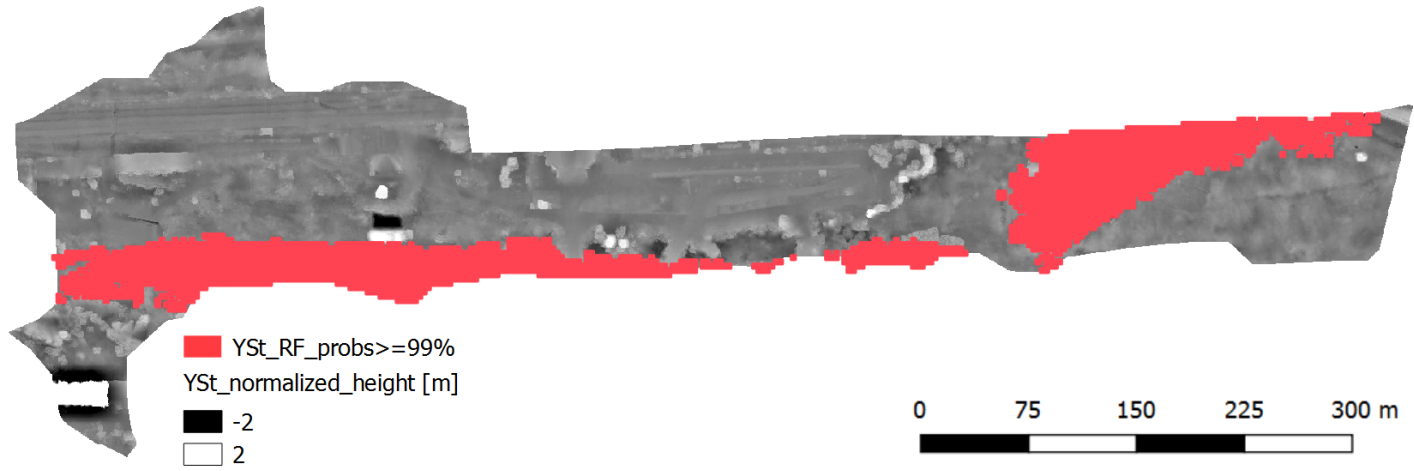

(b)

Figure 6. Study area 3: (a) Normalized height map and local component of the gamma spectrometer measurements [ $\mu \mathrm{Sv} / \mathrm{h}]$; (b) Normalized height map and classification result for class "contaminated", class probability values $>99 \%$

Table 3. Overview of the RF classification parameters and results

\begin{tabular}{|c|c|c|c|}
\hline study area & 1 & 2 & 3 \\
\hline \hline used \#feat & 16 & 12 & 2 \\
\hline class names & $\begin{array}{c}\text { 1: clamp } \\
\text { 2: no clamp }\end{array}$ & $\begin{array}{c}\text { 1: trench } \\
\text { 2: no trench }\end{array}$ & $\begin{array}{c}\text { 1: contaminated } \\
\text { 2: non-cont. }\end{array}$ \\
\hline training samples & 83643 & 2473 & 86862 \\
\hline 5-fold CV & OA 98.8\% & OA 99.8\% & OA 98.5\% \\
(training data) & kappa 94.6\% & kappa 99.6\% & kappa 96.6\% \\
\hline test samples & 52724 & 2446 & 120151 \\
\hline OA [\%] & 95.6 & 99.0 & 98.4 \\
kappa [\%] & 89.5 & 98.0 & 95.5 \\
prec. [\%] & 93.6 & 99.4 & 95.9 \\
rec. [\%] & 91.7 & 98.6 & 97.3 \\
\hline
\end{tabular}

\subsubsection{Study area 3: Yanov Station 3.3}

In the area Yanov Station 3.3, an initial visualization showed a potential correlation between the terrain height anomalies and the local component of the gamma spectrometer measurements. In this study area, the fairly sparse vegetation coverage motivates the usage of the bi-linearly interpolated spectrometer data. The impact of the low vegetation on the measured radiation signal is assumed to be negligible. In other words, we suppose that the gamma spectrometer measurement is a gamma ray superimposition of the buried radioactive material, the constant impact of the top soil layer, and the constant impact of the background radiation from space. Due to lacking reference data a test dataset was manually generated taking into account the information of the normalized height map - overlaid with the local component of the gamma spectrometer measurements (Figure 6a). The selection of features is not necessary on a feature set consisting of only two features (normalized height, gamma_local). The local component of the gamma spectrometer measurements turned out as the five times more important feature in the RF feature impor- tance assessment using the mean decrease in accuracy. The parameters describing the classification quality are very satisfying $(\mathrm{OA}=98.4 \%$, $\mathrm{kappa}=95.5 \%$, precision $=95.9 \%$, recall $=97.3 \%$; see Table 3 ). The prediction result for the study area 3 is presented in Figure $6 \mathrm{~b}$, showing class probability values of more than $99 \%$ for the class "contaminated". In conclusion, in the partly overgrown area of Yanov Station 3.3, the contaminated areas can be automatically detected using the local component of the gamma spectrometer measurements and the normalized height as features.

\section{DISCUSSION}

The classification accuracy of more than 95\% for DTM grid points potentially located in trenches and clamps is fairly high in all study areas (see Table 3). Our concept proves that the UAV-based detection of unknown radioactive biomass deposits in the ChEZ is successful for both multi-spectral data and LiDAR data. The normalized height appears to be the most important feature for the selected areas. Furthermore, the results in study area 2 (Red Forest 2.5) show that the features derived from the $3 \mathrm{D}$ vegetation mapping have also a significant impact on the classification result in forested areas. In non-forested areas like study area 1, the multi-spectral features (i.a. NIR) are important to classify contaminated clamps.

The integration of gamma spectrometry features into the classification process is problematic since the measured radiation is a superposition of different effects. As to be expected, our results verify that bi-linearly interpolated gamma spectrometry data is suitable in study area 3 . Similar results were expected for study area 1 , but it looks like that the use of bi-linearly interpolated gamma spectrometry data is not applicable here 
since the spectrometry features significantly deteriorate the classification result. One explanation might be that the deposits are shielded by a thick soil coverage. A feature assessment in study area 2 (Red Forest 2.5) leads to the assumption that gamma spectrometry data cannot be utilized as bi-linearly interpolated data. Especially in the forested areas, a model with a higher complexity is needed to remove the radiation impact of the above-ground biomass from the gamma spectrometry data and to isolate the radiation impact generated by the buried radioactive material.

An internal technical report about the results of the UAV gamma spectrometry surveys in 2015 (Molitor et al., 2017a) states that burials can be identified by analyzing anomalies of the local component of the gamma intensity. The probability ranges from more than $50 \%$ in vegetated areas to more than $90 \%$ in low vegetated areas. Our results demonstrate that comparable results can be achieved for low vegetated areas just using multi-spectral data (study area 1). However, in vegetated areas (study area 2), a considerable improvement could be reached - as expected - by taking advantage of LiDAR data. Thus, UAV LiDAR is mandatory to detect trenches and clamps in forested areas because of its capability to penetrate vegetation.

\section{CONCLUSION AND OUTLOOK}

The experiments prove that the UAV-based LiDAR and multispectral image technology in combination with aerial gamma spectrometry surveys can successfully map unknown deposits of buried radioactive biomass in the ChEZ. Excellent RF classification results for the detection of trenches and clamps were achieved by fusing geometric features, newly developed vegetation features, gamma spectrometer measurements and multi-spectral image data. To minimize workers dose uptake and to optimize remediation work, the existing hazard maps were verified and upgraded.

Future work will focus on the generation of overlapping datasets combining LiDAR data and multi-spectral image data for overgrown and non-overgrown areas. This data fusion enables the advantages of two sensor technologies in different situations:

- LiDAR data: accurate DTM, LiDAR metrics and tree features in forested areas

- Multi-spectral data: improved classification results, especially in non-forested areas

As far as the methodology is concerned, the feature set shall be supported by adding e.g. point feature histograms (Rusu et al., 2008), additional 3D and 2D features (Weinmann et al., 2014) and shape distributions (Osada et al., 2002) to further improve the classification result. Above all, research is intended to model the impact of the biomass to the gamma spectrometer measurements and to finally dissolve the impacts of the different radiation sources.

\section{ACKNOWLEDGEMENTS}

We would like to thank Dr.-Ing. Norbert Molitor from Plejades $\mathrm{GmbH}$, Oleksandr Tretyak, and his team from the State Central Enterprise for Radioactive Waste Management for their technical support in the Chernobyl Exclusion Zone. We also highly appreciate the supply of the Trinity drone by Quantum Systems GmbH and the piloting by M.Sc. Benjamin Bachmaier and B.Sc. Niclas Purger.

\section{References}

Bugai, D., Skalskyy, A., Dzhepo, S., Kubko, Y., Kashparov, V., Van Meir, N., Stammose, D., Simonucci, C. and Martin-Garin, A., 2012. Radionuclide migration at experimental polygon at red forest waste site in chernobyl zone. part 2: Hydrogeological characterization and groundwater transport modeling. Applied geochemistry 27(7), pp. 1359-1374.

EU, 2014-2017. Support to radioactive waste management in ukraine. https://nuclear. jrc.ec.europa.eu/tipins/contracts/ support-radioactive-waste-management-ukraine. Accessed: 2018-03-28.

Kashparov, V., Yoschenko, V., Levchuk, S., Bugai, D., Van Meir, N., Simonucci, C. and Martin-Garin, A., 2012. Radionuclide migration in the experimental polygon of the red forest waste site in the chernobyl zone-part 1: Characterization of the waste trench, fuel particle transformation processes in soils, biogenic fluxes and effects on biota. Applied geochemistry 27(7), pp. $1348-1358$

Melcher, C. L., 2000. Scintillation crystals for pet. The journal of nuclear medicine 41(6), pp. 1051.

Molitor, N. et al., 2017a. Technical report task d3: Annex 16: Complementary airborne geophysical survey. Unpublished internal technical report.

Molitor, N., Thierfeldt, S., Haneke, K., Nitzsche, O., Bugai, D., Sizov, A. and Drace, Z., 2017b. Recent safety assessment findings on management of legacy wastes from chornobyl accident.

Næsset, E., 2004. Practical large-scale forest stand inventory using a small-footprint airborne scanning laser. Scandinavian Journal of Forest Research 19(2), pp. 164-179.

Osada, R., Funkhouser, T., Chazelle, B. and Dobkin, D., 2002. Shape distributions. ACM Transactions on Graphics (TOG) 21(4), pp. 807-832.

Reitberger, J., Schnörr, C., Krzystek, P. and Stilla, U., 2009. 3D segmentation of single trees exploiting full waveform lidar data. ISPRS Journal of Photogrammetry and Remote Sensing.

Repola, J., Ojansuu, R., Kukkola, M. et al., 2007. Biomass functions for scots pine, norway spruce and birch in finland. Technical report, Working Papers of the Finnish Forest Research Institute 53

Rusu, R. B., Marton, Z. C., Blodow, N. and Beetz, M., 2008. Learning informative point classes for the acquisition of object model maps. In: Control, Automation, Robotics and Vision, 2008. ICARCV 2008. 10th International Conference on, IEEE, pp. 643-650.

Weinmann, M., Jutzi, B. and Mallet, C., 2014. Semantic 3d scene interpretation: a framework combining optimal neighborhood size selection with relevant features. ISPRS Annals of the Photogrammetry, Remote Sensing and Spatial Information Sciences 2(3), pp. 181.

Widlowski, J., Verstraete, M., Pinty, B. and Gobron, N., 2003. Allometric relationships of selected european tree species. Rep. EUR 20855 EN.

Zabulonov, Y., Burtnyak, V. and Odukalets, L., 2017. System for effective remote control and monitoring of radiation situation based on unmanned aerial vehicle. 13, pp. 40-45.

Zabulonov, Y. L., Burtnyak, V. and Zolkin, I., 2015. Airborne gamma spectrometric survey in the chernobyl exclusion zone based on oktokopter uav type. Voprosy Atomnoj Nauki $i$ Tekhniki pp. 163-167. 